\title{
Hematoma subdural crónico. Una actualización y revisión
}

\section{Chronic Subdural Hematoma. An Update and Review}

Recibido: 23 Abril 2020 | Aceptado: 13 Mayo 2021

Juan Carlos Gómez Vega

Médico residente de quinto año de Neurocirugía. Miembro del grupo de investigación Neurología y Neurocirugía, Hospital Universitario San Ignacio, Bogotá, Colombia ORCID: https://orcid.org/0000-0002-4385-5063 Maria Ximena Rojas Q Médica y cirujana, Pontificia Universidad Javeriana, Bogotá, Colombia ORCID: https://orcid.org/0000-0002-1430-6954

a Autora de correspondencia: mrojasq@javeriana.edu.co

Cómo citar: Gómez Vega JC, Rojas MX. Hematoma subdural crónico: una actualización y revisión. Univ. Med. 2021;62(4). https://doi.org/10.11144/Javeriana. umed62-4.hsca

\section{RESUMEN}

El hematoma subdural crónico es una de las entidades más frecuentes en la práctica médica general y neuroquirúrgica, por lo cual su diagnóstico y tratamiento mejora el pronóstico de los pacientes. El factor más frecuentemente implicado para el desarrollo es el trauma; sin embargo, se conocen otros factores como la anticoagulación, la fragilidad o la dependencia funcional total, las comorbilidades, la atrofia cerebral, que también desempeñan un rol importante. El tratamiento del hematoma subdural crónico es quirúrgico. Cuando este está contraindicado, es recurrente o no está indicada la intervención quirúrgica, se plantean otros tratamientos como el manejo médico y la embolización de la arteria meníngea media, el cual ha ganado un gran auge en los últimos años. El objetivo del presente artículo es describir los conceptos generales del hematoma subdural crónico, con énfasis en el manejo médico actual y la terapia endovascular.

\section{Palabras clave}

embolización; hematoma subdural crónico; recurrencia; arterias meníngeas; craneotomía.

\footnotetext{
ABSTRACT

Chronic subdural hematoma is one of the most frequent entities in neurosurgical practice, therefore, its diagnosis and treatment improves the prognosis of patients. The most frequent factor involved is trauma, although, other factors as anticoagulation, comorbidities and cerebral atrophy may also play an important role. Historically, surgical treatment has been the treatment of choice, however, when it is recurrent, other treatments as embolization of the middle meningeal artery are proposed. The aim of this article is to describe the general concepts of chronic subdural hematoma with an emphasis on the embolization of the middle meningeal artery as a treatment for chronic recurrent subdural hematoma. No conflicts of interest are declared in this review.

Keywords

embolization; chronic subdural hematoma; recurrence; meningeal arteries; craniotomy.
} 


\section{Historia}

El hematoma subdural crónico (HSDc) fue documentado por el médico alemán Johann J. Wepfer en 1657, cuando al realizar una necropsia de un paciente con sospecha de accidente cerebrovascular, encontró un quiste subdural lleno de sangre, lo cual podía corresponder a un hematoma subdural $(1,2)$. Sin embargo, se le atribuye la descripción formal al Dr. Virchow, en 1857, el cual lo llamó una paquimeningitis hemorrágica, ya que se pensaba que ocurría por inflamación de la duramadre. En el siglo XX se comenzó a aceptar la etiología traumática como la más común de esta entidad (1).

El término fue continuado por Putman y Cushing, en 1925, y fue apoyado en 1972 por Watanabe et al. (3), quienes crearon el primer modelo animal, introduciendo líquido cefalorraquídeo y sangre coagulada en el espacio subdural en perros y monos. Posteriormente, en 1974, este modelo fue controvertido, dado que se planteó que no era necesario la presencia de líquido cefalorraquídeo para formar membranas características del hematoma subdural, sino que se creaba tanto una cápsula biológica a partir de la función de la fibrina y el tejido granuloso como una hemorragia recurrente (2). A partir de esto, comenzaron diferentes hipótesis respecto de lo que podría causar un HSDc, las cuales hasta el día de hoy siguen siendo profundamente estudiadas.

\section{Epidemiología}

El HSDc es más frecuentemente en pacientes adultos entre la quinta y séptima décadas de la vida (usualmente en mayores de 65 años), con una incidencia de 58,1 por cada 100.000 personas al año. La incidencia en la población general es de 1 a 5 casos por cada 100.000 personas al año $(1,4)$. Se presenta con mayor frecuencia en el género masculino que en el femenino, en una proporción del $77 \%$, en comparación con el $23 \%$, respectivamente (5). El HSDc recurrente puede definirse con criterios clínicos y radiológicos. Desde el punto de vista clínico, se considera recurrencia cuando después de una mejoría y un tratamiento adecuado, el paciente presenta estado mental alterado, empeoramiento de la condición neurológica preexistetente, déficit focal de reciente inicio o cefalea sin déficit focal necesario concomitante (6). La recurrencia radiológica es descrita como el crecimiento o recidiva posoperatoria dentro de los primeros 6 meses, por lo general antes de 3 meses, que genera síntomas neurológicos, aumento de volumen del espacio subdural en el lado que fue tratado en comparación con el volumen medido 1-3 días del posoperatorio y la compresión del cerebro por un nuevo hematoma subdural observado en la tomografía axial computarizada (TAC) de cráneo 3 meses después de la cirugía (6-8). Esto, en ocasiones, se da por factores predisponentes, inherentes al paciente, como sus comorbilidades y su edad avanzada, o por causa del procedimiento, por ejemplo, cantidad de sangre drenada, aire dejado en el lecho quirúrgico, entre otros (8-10). Se ha demostrado que la recurrencia es también mayor en hombres, con un $73,8 \%$, en relación con un $26,2 \%$ en el caso de las mujeres $(5,11)$. Durante años, el manejo quirúrgico de elección para el tratamiento de los hematomas subdurales fue el drenaje mediante craneotomía con agujeros con trépano del cráneo. Con este manejo se ha reportado una recurrencia que puede llegar a ser del $11-28 \%$, y a pesar de reintervenirse el paciente, en ocasiones puede volver a presentar recurrencia $(12-15)$.

\section{Factores de riesgo}

Dentro de los factores de riesgo más relevantes se encuentran la edad avanzada (11-37\%), por la atrofia cerebral y la fragilidad de las venas; el alcoholismo (15,6\%); las convulsiones (3,5\%); procedimientos como derivaciones ventriculoperitoneales $(0,9 \%)$, y punciones lumbares. Adicionalmente, las comorbilidades del paciente, por ejemplo, malformaciones vasculares $(0,4 \%)$, quistes aracnoideos $(11,1$ $\%)$, enfermedades cerebrovasculares $(4,5 \%)$, hipertensión arterial $(20,5 \%)$, cardiopatía $(35,5$ $\%)$, hemodiálisis, traumatismos repetidos $\mathrm{y}$ 
coagulopatías aumentan el riesgo de presentar recurrencia $(1,10-12,16,17)$. Es claro que la anticoagulación con antagonistas de la vitamina $\mathrm{K}$ o los nuevos anticoagulantes orales como el dabigatrán (4 veces mayor el riesgo que la población general) — o la ingesta de antiplaquetarios — como la aspirina- y simpaticomiméticos — como la seudoefedrinaaumentan el riesgo de recurrencia del hematoma $(18,5 \%)(13,16-18)$.

\section{Fisiopatología}

Se han propuesto dos teorías para la formación de los HSDc (1). La primera, descrita como la teoría del gradiente osmótico, propuesta por Gardner, en 1932, afirma que el aumento de proteínas en el hematoma cambiaba la presión osmótica y hacía que el fluido entre en mayor cantidad hacia el interior del hematoma, lo cual incentiva su crecimiento (1). Posteriormente, se demostró que el contenido de un hematoma es isosmótico al líquido cefalorraquídeo y a la sangre. La segunda teoría se basa en estudios histológicos en los cuales se evidenció la existencia de eritrocitos frescos, y con ello se postula que el hematoma lo forma una hemorragia recurrente que proviene de la cápsula con hiperfibrinólisis. En este último proceso participan enzimas que generan estimulación celular y angiogénesis, como el factor de crecimiento vascular endotelial (VEGF) y mediadores inflamatorios como la bradicinina, la calicreína y el factor actividad de plaquetas, todos estos encargados de aumentar la permeabilidad vascular y la vasodilatación, al liberar un activador tisular de plasminógeno, lo que predispone al resangrado crónico (1).

Microscópicamente, los HSDc tienen dos neomembranas, la visceral y la parietal. La primera es la más interna, la más delgada y surge de la escisión formada del borde dural. Entre tanto, la parietal es la membrana externa, con elementos que no se presentan en la histología normal. Se genera entonces un clivaje de esta membrana que lleva a la aparición de neovasos, fibras de colágeno y elastina, miofibroblastos, sustancias inflamatorias como
IL-6, IL-8, $\alpha$-TNF y cuerpos de Weibel-Palade. Es esa neovascularización la encargada de generar una hemorragia recurrente con crecimiento del HSDc (19).

Así mismo, la estructura de estos capilares es anormal, debido a que se organizan en grandes sinusoides con paredes frágiles que presentan uniones intracelulares débiles y enzimas fibrinolíticas que contribuyen al proceso de hemorragia. Por ello, la membrana externa de los hematomas se cree es la responsable de la evolución del mismo hematoma, que ha sido comprobada por imágenes de microscopía avanzada y biología molecular. Esto da el punto de partida de las medidas terapéuticas para el tratamiento no quirúrgico, como los corticoesteroides, los antifibrinolíticos, las estatinas o los inhibidores de la enzima convertidora de angiotensina, que buscan inhibir las sustancias que promueven la fibrinolisis, la ruptura de las capas durales y el sangrado recurrente; sin embargo, este tipo de tratamientos no ha mostrado una eficacia real en todas las poblaciones, sino en poblaciones específicas $(20,21)$.

Sumado a lo anterior, histológicamente se han descrito pequeñas arterias comunicantes provenientes de la arteria meníngea media que penetran la dura y se conectan con la neovascularización de la membrana externa. Esta razón respalda el uso de la embolización de la arteria meníngea media, porque inhibe el influjo de sangre $y$, por ende, promueve la resolución y el control de la hemorragia $(19,22-24)$.

\section{Clínica}

La expansión gradual del hematoma es la responsable de generar los síntomas de los pacientes, quienes, en principio, pueden llegar a ser asintomáticos, por el poco efecto compresivo de masa que generan estos hematomas subdurales; pero que, al avanzar en el tiempo, y por ende, en la cantidad de volumen recolectada, pueden presentar alteraciones del estado de conciencia, como síntoma cardinal en esta patología (1). 
El deterioro cognitivo y los cambios comportamentales se acompañan de otros síntomas, como cefalea crónica e intensa, en raras ocasiones alteración del lenguaje o de la marcha, hemiparesia y hasta convulsiones $(1,2,25)$. Todos estos síntomas no se producen abruptamente, sino de manera progresiva, al parecer explicados por la capacidad del cerebro de acoplarse a los cambios de presión y volumen secundarios a un curso lento y gradual de aumento del hematoma subdural, lo que lleva a que no haya un síndrome de hipertensión endocraneana abrupto. Si todo progresa, el paciente puede llegar al estado de coma por un deterioro rostrocaudal.

En cuanto a los estadios clínicos de un HSDc, se clasifica de dos maneras: por sintomatología clínica y por su temporalidad. En cuanto a esta última, los HSDc se clasifican en agudo, que es aquel que se presenta desde el inicio hasta el tercer día; el subagudo, del día 4 al 13, y el crónico, después del día 14 del inicio (26). Por otro lado, en cuanto a sintomatología clínica, por medio de la Escala de Markwalder (27), se plantean cuatro grados de clasificación de acuerdo con la clínica de ingreso del paciente: el grado 0 , cuando el paciente está asintomático con examen neurológico normal y con diagnóstico solamente imagenológico. Un grado I, en el que el paciente tiene una Escala de Coma de Glasgow (GCS) de 15, sin déficit neurológico pero con síntomas leves como cefalea o marcha inestable. El grado II, aquel en el que existe una escala GCS de 14-13 o con déficit neurológico variable como hemiparesia. El grado III, con una GCS de 12-9, en el que el paciente se encuentra estuporoso pero responde a estímulos dolorosos, con signos focales graves como hemiplejía. Finalmente, un grado IV, con una GCS menor a 9, con el paciente comatoso o con ausencia de respuesta a estímulos, que genera decerebración y decorticación $(28,29)$.

\section{Diagnóstico imagenológico}

\section{Tomografía axial computarizada}

Dado que el cuadro clínico puede simular cualquier entidad neuroquirúrgica, es necesario complementar con un diagnóstico imagenológico a través de la TAC de cráneo, que servirá no solo para confirmar el diagnóstico, sino para conocer su localización, volumen, compresión al parénquima cerebral y tiempo de evolución. Esto último es relevante a la hora de escoger el abordaje para el paciente, ya que un hematoma subdural que sea de inicio agudo se verá hiperdenso (70-90 HU) y aquel que sea subagudo será isodenso (28-48 HU). Estos son los más complicados de identificar, por su similitud con el parénquima cerebral. Finalmente, los HSDc serán hipodensos (8-28 HU), según se muestra en la figura $1(2,29)$.

\section{Figura 1.}

Tipos de hematomas subdurales de acuerdo con la temporalidad

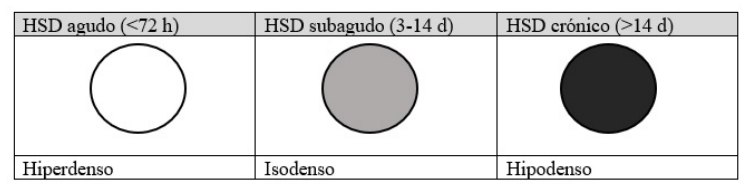

Resonancia magnética cerebral

Si bien la TAC es la imagen más utilizada y con la que rápidamente se puede lograr un diagnóstico, no va a superar en sensibilidad a una resonancia magnética cerebral. A su vez, permite identificar con mayor especificidad los estadios en temporalidad de los hematomas, de acuerdo con los cambios en la condición de la hemoglobina, como se enumeran en la tabla $1(30,31)$. 
Tabla 1.

Cambios del hematoma subdural con tomografía de cráneo y resonancia magnética cerebral según las condiciones de hemoglobina por tiempo de evolución (30)

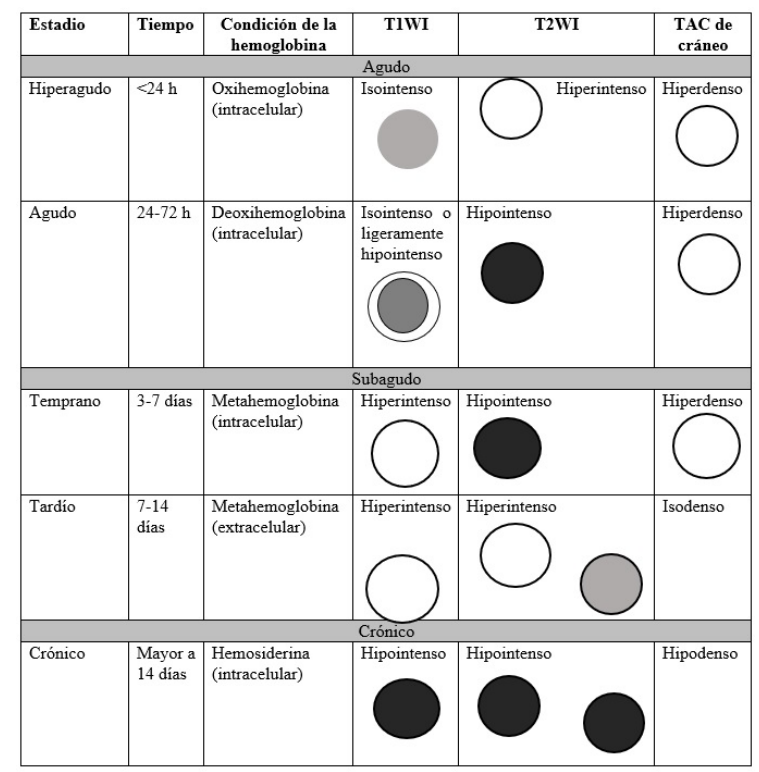

Como se mencionó, la resonancia magnética cerebral identifica en mayor proporción y con mayor caracterización los hematomas subdurales y su temporalidad. Esto se logró comprobar en un estudio realizado a 34 pacientes intervenidos quirúrgicamente para drenaje de un HSDc. En el $60 \%$ de los casos se identificó que era recurrente, porque estaba encapsulado; mientras que solo el $27 \%$ de estas fue identificada mediante TAC $(2,32,33)$. Sin embargo, esta última sigue usándose como abordaje inicial por encima de la resonancia magnética cerebral, ya que tendrá mayor disponibilidad en diferentes niveles de atención, implicará menos costo y será más rápida, sobre todo para abordar pacientes que lleguen posterior a traumatismos.

Es menester recordar que en una resonancia magnética un HSDc organizado se verá hipointenso en T1 y T2, al igual que pasaría en una tomografía, como se evidencia en la tabla 1. Esto se debe a que los hematomas, a medida que pasa el tiempo de evolución, generan neomembranas que pueden llevar a encapsular el hematoma y, por ende, en dicho estadio habría muy poca probabilidad de que un hematoma ya organizado se pueda resolver solamente con un abordaje de drenaje quirúrgicamente, pues en muchas ocasiones se va a necesitar una escisión de la cápsula ya formada (34).

Existen factores de riesgo imagenológico que se correlacionan con una mayor proporción de resangrado o reaparición del hematoma. Dentro de estos se ha visto la reexpansión del parénquima cerebral, donde se localizaba el hematoma subdural después de una semana de la cirugía, ya que hay mayor recurrencia si el espacio subdural sigue siendo mayor a $10 \mathrm{~mm}$ por poca reexpansión $(13,3 \%)$; pero también un drenaje rápido puede producir el efecto contrario, porque aumenta el riesgo de recurrencia por una acumulación masiva de aire $(23,8 \%)$, por la desviación de la línea media mayor a 5-10 mm $(14,5 \%)$, por un hematoma subdural que afecte el lóbulo frontal, por la presencia de coágulos subdurales agudos en el hematoma subdural 4 días después de la cirugía, por tener apariencia de estar separado o loculado y no homogéneo (25 $\%)$, entre otros $(2,6,7,10,34)$.

\section{Angiografía cerebral}

$\mathrm{Al}$ realizar una angiografía se ha demostrado que hay una dilatación anormal difusa de la arteria meníngea media con redes de neovascularización que se conectan con los capilares formados de la cápsula externa del hematoma subdural, lo cual apoyaría una de las teorías fisiopatológicas con mayor representación (18).

\section{Diagnósticos diferenciales}

Dado que uno de los primeros síntomas es la alteración del estado de conciencia, con alteración secundaria del comportamiento y deterioro cognitivo, al presentarse en mayor frecuencia en poblaciones de edad avanzada, se puede confundir con una demencia (25). Cuando el paciente acude al servicio de urgencias, deben descartarse los eventos cerebrovasculares agudos, bien sea isquémicos o hemorrágicos, ya que hasta no tener una neuroimagen confirmatoria, la clínica de alteración del estado de conciencia, 
la alteración motora y la alteración del lenguaje llevarían a considerarlos como diagnóstico diferencial.

\section{Tratamiento}

\section{Médico}

El tratamiento de los HSDc depende del estado clínico inicial o de ingreso del paciente, por ejemplo, el estado de conciencia de acuerdo con la escala GCS, sumado a factores que permitirán un adecuado seguimiento como posibilidad de control continuo del paciente y su asistencia a las citas, acompañamiento continuo en caso de ser de avanzada edad, entre otros factores. Dependiendo de esto, el tratamiento puede ser desde observación médica del paciente, hasta tratamiento no quirúrgico y quirúrgico.

En primer lugar, el tratamiento conservador ha mostrado una baja tasa de éxito (3-18\%) (4). Como se había mencionado en el apartado de la fisiopatología, se cree que toda fuerza que genere retracción del parénquima cerebral y aumento secundario del espacio subdural aumenta el riesgo de progresión del hematoma subdural, incluidas las comorbilidades previas de los pacientes y la madurez de las neomembranas; por el contrario, factores que mejoren la expansión del parénquima cerebral promueven la resolución espontánea $(35,36)$. De esta forma, para aquellos pacientes que no tienen síntomas o son muy leves, o tienen comorbilidades que no permiten ser operados, se sugiere el tratamiento conservador con seguimiento con TAC de cráneo.

En cuanto al tratamiento médico no quirúrgico del HSDc, se han utilizado diferentes medicamentos como inhibidores de la enzima convertidora de angiotensina, estatinas, medicamentos antifibrinolíticos y corticoesteroides.

\section{Esteroides}

El fundamento de utilizar medicamentos como los corticoesteroides se basa en que estos inhiben la síntesis de mediadores proinflamatorios, el reclutamiento de células del sistema inmune, el óxido nítrico y la ciclooxigenasa, y sobre todo inhiben la síntesis del VEGF, el cual aumenta la permeabilidad vascular (36-38).

Inicialmente, los diferentes estudios acerca del uso de corticoesteroides en los HSDc recomendaban el uso de dexametasona, pues garantizaba adecuados desenlaces en los pacientes y una estadía hospitalaria no tan prolongada; al igual que ocurría con pacientes que eran sometidos a tratamiento de drenaje quirúrgico del hematoma. En su estudio, Thotakura et al. (39) deciden proponer un sistema de clasificación radiológica (AmitRao Radiologic Grading of Chronic Subdural Hematoma) para identificar qué pacientes podrían ser tratados con corticoesteroides; de este modo, clasificaron a los pacientes de acuerdo con el tamaño del hematoma con la desviación de la línea media: pequeño, cuando no desvía la línea media; intermedio, cuando la desvía en menos de $5 \mathrm{~mm}$, lo que da un punto; grande, al desviar de 5 a $10 \mathrm{~mm}$, lo que otorga 2 puntos, y masivo, cuando desvía más de $10 \mathrm{~mm}$ y da 3 puntos. Como segundo ítem, tuvieron en cuenta la densidad basada en las unidades Hounsfield en la TAC, lo que divide la densidad en menor a 30 , de $31-40$ o mayor de 40 , y otorga 0,1 y 2 puntos, respectivamente. $\mathrm{Al}$ ser bilateral, se aumentaba un punto adicional. De este modo, cuando los pacientes tienen hematomas considerados de bajo grado (0-2), el uso de corticoesteroides sería más beneficioso que en pacientes con altos grados en esta clasificación (4 y 5).

En estudios realizados en pacientes con HSDc, la colocación de esteroides con uso de la dexametasona en el preoperatorio con una dosis intravenosa de $4 \mathrm{mg}$ cada 8 horas durante 3-4 días; luego, un destete paulatino de $2 \mathrm{mg}$ por 3 días y $1 \mathrm{mg}$ por 3 días más disminuyó la tasa de recurrencia en el posoperatorio y se asoció con mayor tasa 
de supervivencia y no se demostró mayor incidencia de complicaciones ni de mortalidad (28,37,39-41). En contraposición, en un ensayo clínico multicéntrico aleatorizado realizado en el Reino Unido por Hutchinson et al. (42), al comparar pacientes que habían sido operados inicialmente y tratados con dexametasona oral $8 \mathrm{mg} 2$ veces al día posteriormente con un grupo placebo, se evidenció que aquellos tratados con dexametasona tuvieron menos desenlaces favorables, entendido esto como una Escala de Rankin de $0-3$ a los 6 meses, que aquellos del grupo placebo (84\% y $90 \%$, respectivamente). Sin embargo, tuvieron menos necesidad de repetir procedimientos quirúrgicos que el placebo (2\% y $7 \%$, respectivamente).

Inhibidores de la enzima convertidora de angiotensina

Los inhibidores de la enzima convertidora de angiotensina se han usado basándose en su mecanismo de funcionamiento para disminuir la actividad angiogénica e inflamatoria del VEGF en ciertas patologías (como retinopatía diabética y síndrome de Kaposi), por lo que se cree también que ayuda disminuyendo el riesgo de recurrencia del HSDc $(36,43)$. Sin embargo, posteriormente en un estudio aleatorizado realizado por Poulsen et al. (44), utilizando $5 \mathrm{mg}$ al día de perindopril 3 meses previos a la cirugía, no se evidenció disminución del volumen de HSDc 6 semanas después del procedimiento quirúrgico y tampoco disminuyó su recurrencia. Se ha considerado que esto acaece, dado que los inhibidores de la enzima convertidora de angiotensina aumentan las concentraciones de bradiquinina, que es un péptido vasoactivo, capaz de aumentar la vasodilatación y la permeabilidad, es decir, la extravasación de sangre de las membranas al HSDc. Por esta razón, en un estudio reciente de Neidert et al. (45), el volumen del HSD en los pacientes con inhibidores de enzima convertidora de angiotensina fue mayor que aquellos que no recibían dicho procedimiento. Según varios estudios, no disminuyen la recurrencia de hematomas (43-46).

\section{Antifibrinolíticos}

Otro de los tratamientos médicos que se han contemplado es el ácido tranexámico, que tiene un efecto antifibrinolítico, es decir, que inhibe la actividad del plasminógeno y de la plasmina, y logra de este modo que se restrinja la hiperactividad fibrinolítica y con ello la permeabilidad vascular del hematoma, los microsangrados. Ello contribuye a su absorción $(36,46)$. Uno de los primeros estudios realizados en este campo fue en una población de pacientes en hemodiálisis con HSDc refractario a tres intervenciones quirúrgicas, donde se les administró ácido tranexámico intravenoso a dosis de $20 \mathrm{mg} / \mathrm{kg}$ cada $48 \mathrm{~h}$ durante 4 semanas y luego se les redujo la dosis a la mitad vía oral por otras 4 semanas. Con ello se logró la resolución radiológica del hematoma (47).

En cuanto al ácido tranexámico oral, en un estudio retrospectivo de Kageyama et al. (48) y de Tanweer et al. (49) se aplicaron dosis de $750 \mathrm{mg}$ al día durante 1-4,5 meses y de 650 $\mathrm{mg}$ al día durante un mes, respectivamente. Ello disminuyó la recurrencia del HSDc y funcionó como terapia adyuvante. La aplicación de ácido tranexámico todavía es un tema controversial, dado que no se ha estudiado su aplicabilidad en pacientes anticoagulados por riesgo aumentado de efectos trombóticos y porque no se conoce su efecto en la reabsorción del hematoma. Un ensayo clínico doble ciego que sigue en marcha, TRACS (50), ha demostrado la efectividad de este tratamiento en 22 pacientes; sin embargo, se esperan los resultados finales.

\section{Antagonista del factor activador de plaquetas}

Se ha demostrado que el antagonista del factor activador de plaquetas es un potente mediador de lípidos en inflamación, los cuales desempeñan un rol importante en la formación y crecimiento de la membrana de HSDc. En ciertos estudios se ha demostrado que estos medicamentos funcionan para reducir la necesidad de cirugía y la recurrencia posterior a esta, empleando 3 mg al día de etizolam 14 días antes de la cirugía 
(51). Así se aplicó en el estudio de Hirashima et al. (51), dado que el etizolam es un ansiolítico análogo de las benzodiacepinas que actúa como inhibidor del factor activador de plaquetas. En el grupo en el que se aplicó se demostró una ausencia de la recurrencia a los 3 meses y una disminución del volumen del hematoma en la tomografía al mes, que fue estadísticamente significativa con respecto al grupo control. Sobre todo se ha logrado comprobar que este tipo de medicamentos funciona cuando los hematomas se encuentran en estadios de Higromas.

\section{Terapia osmótica}

Existen otras alternativas como lo son el manitol, que se utiliza basándose en que los hematomas subdurales aumentan su tamaño por el gradiente osmótico que existe adentro. A su vez, a medida que va aumentando su tamaño, genera una tensión tal en la cápsula que induce los microsangrados. En este orden de ideas, al lograr disminuir la presión interna usando manitol, se puede evitar esta cadena de eventos $(36,52)$. Sin embargo, los hallazgos en cuanto al tema del manitol son también controversiales: en un estudio publicado en 1970, de Suzuki y Takaku (53), al administrar manitol al $20 \%, 1000 \mathrm{~cm}^{3}$ al día, se demostró la resolución completa del HSDc en 22 pacientes. Caso contrario ocurrió en el estudio de Gjerris y Schmidt (54) años después, en el que también se utilizó manitol al 20 $\%, 1000 \mathrm{~cm}^{3}$ en 4-6 horas diariamente, pues los autores concluyeron que dicho tratamiento no puede remplazar el manejo quirúrgico, dado que en algunos pacientes avanzó la sintomatología neurológica o no hubo cambio alguno en el tamaño de hematoma en la arteriografía realizada a los 20 días de control. En todos los casos se requirió cirugía.

\section{Estatinas}

El uso de las estatinas como la atorvastatina se basa en el principio de su inhibición de la inflamación y la promoción de la angiogénesis. De este modo, se demostraba que la recuperación de los pacientes en los que las estatinas se usan como terapia coadyuvante sería más rápida y en un alto volumen de pacientes. En un estudio realizado por Wang et al. (55) se demuestra que los pacientes que recibieron una dosis de atorvastatina a $20 \mathrm{mg}$ una vez al día entre 1 y 6 meses pueden presentar la resolución casi que total y notoria en estudios de neuroimágenes del HSDc y, así mismo, mejora el estado neurológico del paciente. Esto se ve aproximadamente al mes de la lesión, y a los 6 meses por lo general no existe hematoma o ha desaparecido.

\section{Quirúrgico}

En cuanto al tratamiento quirúrgico, existen diferentes técnicas para realizar el abordaje, y la decisión de llevar al paciente a dicho procedimiento o no se basa en las indicaciones principalmente clínicas que, de acuerdo con Bullock et al. (56), son que todo paciente con una GCS $<9$ debe recibir monitorización de la presión intracraneal. Los hallazgos radiológicos en neuroimagen de un tamaño del HSDc tal que genera desplazamiento de la línea media mayor a $5 \mathrm{~mm}$ o un volumen del hematoma mayor a $10 \mathrm{~mm}$ deben tratarse de manera quirúrgica, y aquellos pacientes que presentan una GCS $<9$ sin estos hallazgos en TAC cerebral solo serán manejados de manera quirúrgica en caso de que presenten un deterioro del estado de conciencia con una GCS con disminución de 2 puntos desde la admisión al hospital, anisocoria o midriasis y presión intracraneal que exceda los $20 \mathrm{mmHg}$ (57-59).

Esta decisión también se basa en cada caso clínico particular, es decir, en las comorbilidades del paciente, medicamentos con los que está en tratamiento y, sobre todo, edad y dependencia funcional, con el fin de equilibrar el riesgobeneficio para cada persona, ya que en muchas ocasiones el hecho de tener una edad avanzada (>75 años), comorbilidades cardiovasculares que aumenten el riesgo del procedimiento y la anestesia o un estado neurológico prácticamente irreversible contraindica el procedimiento quirúrgico $(56,57)$. 
Las técnicas quirúrgicas usuales son tres: craneostomía con taladro giratorio (twist drill), craneostomía con agujeros de trepanación (burr hole) y craneotomía (craneotomy) (59). Respecto a la craneostomía con taladro giratorio, se trata de la creación de un agujero en la parte de mayor grosor del HSDc y la colocación posterior de un sistema de drenaje cerrado, $20 \mathrm{~cm}$ por debajo de agujero de la craneostomía, el cual se retira cuando el paciente presenta mejoría clínica o al menos se ha drenado el $20 \%$ de la acumulación, siendo un promedio de 1-7 días $(57,59,60)$. Este procedimiento genera un descompresión hemisférica más lenta que otros tipos de procedimientos, con el fin de disminuir complicaciones como la hemorragia. Otra ventaja importante es que puede realizarse bajo anestesia local, lo que evita las complicaciones de una anestesia general y, por ende, es una de las técnicas de elección en pacientes ancianos con múltiples comorbilidades $(57,59,61)$.

En cuanto a la craneostomía con agujeros de trepanación, consiste en la realización de 1 o 2 agujeros de $10-30 \mathrm{~mm}$, por medio de la trepanación del cráneo, en localizaciones específicas de la convexidad, exactamente en el punto donde el hematoma tiene mayor grosor, por lo general a $5-8 \mathrm{~cm}$ de distancia para poder realizar una durostomía, el drenaje del hematoma y la irrigación suave con solución fisiológica mediante sonda hasta obtener líquido claro, para asegurarse de un adecuado drenaje del hematoma $(57,59)$. Por lo general, está recomendada la colocación de un dren por dicha incisión, que se dejará durante aproximadamente $48 \mathrm{~h}$ posoperatorias, para permitir continuar el drenaje del hematoma y la expansión del cerebro, que disminuirá la necesidad de un 10\%-19\% de repetir la cirugía $(24,56,57)$. Esta técnica, de acuerdo con las guías canadienses y británicas, es la más utilizada y la primera línea de manejo quirúrgico, dado que cuenta con un riesgo de recurrencia menor y la mortalidad a los 6 meses desciende del 18,1\% al 9,6\%, al utilizar el dren posterior a la cirugía (62).

La tercera técnica, que es la craneotomía, en la cual se realiza un colgajo óseo mayor a $30 \mathrm{~mm}$, con irrigación y evacuación del
HSDc. Era la técnica de elección antes de la existencia de la TAC, debido a que permitía mayor exposición al neurocirujano; sin embargo, a medida que pasa el tiempo, se ha preferido la microcraneotomía (59). Esta técnica es la indicada en el momento de enfrentarse con HSDc recurrrentes con organizaciones extensas o formación de membranas, es decir, que persisten a pesar de los anteriores procedimientos, o como la primera técnica quirúrgica para pacientes con HSDc, que tiene algún componente agudo $(57,63,64)$.

Existe cierta tasa de recurrencia de los hematomas subdurales. La temprana se define como la reaparición del hematoma que genera síntomas en los primeros 3 meses tras la cirugía del HSDc, y la tardía, cuando supera estos 3 meses (37). Según el análisis multivariado realizado por Moreno Huertas (65), los factores de riesgo que, se ha visto, aumentan la posibilidad de recurrencia de los hematomas son un volumen prequirúrgico del hematoma mayor a $60 \mathrm{~cm}^{3}$ en promedio (OR: 1,01; IC95 \%: 1,00-1,02), una desviación de la línea media $>10 \mathrm{~mm}(\mathrm{OR}$ : 3,03; IC95 \%: 1,02-5,93), una obliteración de cisternas de la base como signos de aumento de la presión intracraneal (OR: 3,95; IC95 \%: $1,13-4,84)$, una arquitectura trabeculada (OR: 3,30; IC $95 \%$ : 1,18-9,03), un neumoencéfalo a tensión (OR: 4.4; IC95\%: 3,68-4,9); mientras que la bilateralidad de los hematomas mostró significancia en el análisis bivariado, mas no en el multivariado (OR: 2,51; IC95 \%: 0,85-7,47). De acuerdo con Kwon et al. (66), al analizar 175 pacientes, se evidenció mayor recurrencia en aquellos que tuvieron un drenaje menor a 200 $\mathrm{cm}^{3}$ en la subdurostomía, que los que tuvieron mayor a esta cantidad; por eso, se espera que durante el tiempo que está la subdurostomía se tenga más de $100-150 \mathrm{~cm}^{3}$ de drenaje para contemplar una menor recurrencia $(8,65,66)$.

Esto hace que se sigan técnicas de tratamiento diferentes o adicionales a las ya utilizadas en el HSDc, como el drenaje de succión subgaleal, que permite un drenaje continuo del hematoma restante. El cierre simultáneo de la fístula dural en el momento de la intervención es un punto óptimo para contrarrestar la recurrencia, incluso 
la administración de al menos $2000 \mathrm{~cm}^{3}$ de líquidos endovenosos en los primeros 3 días posoperatorios asociada con una recurrencia reducida (38). El drenaje subgaleal consiste en la colocación de un tubo de Jackson-Pratt en el espacio subgaleal, con la punta del catéter sobre el trépano realizado, exteriorizado por una incisión en el cuero cabelludo y conectado a un drenaje cerrado para monitorizar la cantidad de drenaje 48-72 h en el posoperatorio (67). Este método de drenaje ha evidenciado una recurrencia del 33,3\%, en comparación con la subdurostomía, que evidenció recurrencia del $24,5 \%$, y el drenaje subdurosubgaleal, que fue del $15,4 \%(68)$.

\section{Terapia endovascular}

La terapia endovascular consiste en la embolización de la arteria meníngea media, que elimina el aporte sanguíneo del HSDc. Dentro de las indicaciones de la terapia endovascular, las tres poblaciones que potencialmente se pueden beneficiar son: primero, los pacientes con hematoma subdural crónico de novo que requirieron manejo quirúrgico, siendo la embolización una terapia adyuvante; segundo, aquellos en quienes falló el tratamiento conservador, y, tercero, aquellos que ya fueron manejados quirúrgicamente $y$, sin embargo, presentan hematoma subdural recurrente (24).

Existen diferentes técnicas para la embolización de la arteria meníngea media, entre ellas, la vía transfemoral, que es la más utilizada, o la transradial o la transcarotídea, menos usadas, y solo cuando la primera no se pueda emplear por comorbilidades del paciente o por lesiones (57). Cuando existe más de un vaso meníngeo como aporte al hematoma, cada uno se debe embolizar $(52,53)$.

Las sustancias utilizadas se dividen en varios grupos: las partículas (por ejemplo, el alcohol polivinílico), debido a que contienen diferentes tamaños y pueden, de este modo, adaptar el nivel de embolización; además, generan una obstrucción mecánica directa que induce una reacción a cuerpo extraño, con efecto permanente. Las esponjas de gelatina como el gelfoam, que no se recomiendan tanto para el tratamiento de HSDc, pues su efecto es transitorio, ya que son reabsorbidas entre las 3 semanas y los 3 meses posteriores. El NButil-2-cianoacrilato, un tipo de cianoacrilatos, que actúan como agente embólico líquido adhesivo que ocasiona una respuesta inflamatoria aguda en los vasos y su oclusión permanente, también recomendado, al igual que alcohol polivinílico, aunque contraindicado en caso de reacción alérgica conocida o existencia de vasoespasmo. Finalmente, el onyx o etilenvinil-alcohol/dimetil sulfóxido, que es un agente no degradable ni adhesivo, biocompatible, que es de elección, pues al hacer contacto con una solución acuosa se precipita en masa esponjosa de manera controlada y es apto para embolizaciones distales. Es preciso tener en cuenta las contraindicaciones en pacientes con falla renal o hepática, antecedente de existencia de vasoespasmo o inestabilidad del catéter (69-72).

En el estudio realizado por Hirai et al. (73) se demostró, por medio de una imagen tomográfica, que posteriormente a la embolización se incrementó la densidad del hematoma, lo que sugiere la extravasación del medio de contraste de la arteria cerebral media por medio de los microcapilares a la cavidad interna del hematoma, lo que apoya la utilidad de este procedimiento en impedir el aporte al hematoma $y$, de este modo, no solamente ayuda en su resolución, sino también en disminuir su recurrencia.

En una serie de 60 casos, Link et al. (13) embolizaron la arteria meníngea media en diferentes contextos: en 42 pacientes, como tratamiento inicial; en 8 pacientes, para la recurrencia de hematoma subdural, y en 10 pacientes, como profilaxis poco después del drenaje quirúrgico. Así, 4 de los 50 casos no profilácticos $(8,9 \%)$ tuvieron una recidiva que requirió drenaje quirúrgico posterior y 31 casos $(68.9 \%)$ tuvieron una resolución o reducción de más del $50 \%$ en el seguimiento. Ello los llevó a tener una tasa de éxito a largo plazo de $91 \%$, por 
lo que amerita se sigan realizando ensayos clínicos a gran escala.

Esto mismo fue apoyado por otros estudios en los cuales también se comprobó una tasa de éxito de rango parecido (90\%); sin embargo, esto se veía afectado por determinantes como que el hematoma estuviera organizado o se hubiera desarrollado circulación colateral que puede disminuir la eficacia. Por esta razón, se recomienda la resonancia magnética cerebral antes de la embolización, para comprobar la organización del hematoma (74). Existen algunos otros factores que no son los más frecuentes, pero es preciso considerar para pensar que un paciente se beneficia del procedimiento de embolización, como lo es el reconocimiento de variantes anatómicas, por ejemplo, que la arteria meníngea media se origine de la arteria basilar, debido a que puede tener más riesgo de reflujo y eventos tromboembólicos a nivel de esta arteria, así como ruptura del vaso. Por tal motivo se recomienda la toma de una angiografía previa al procedimiento (75).

Así mismo, muchos estudios han tenido el objetivo de comparar no solo los desenlaces primarios, como la recurrencia del hematoma subdural, sino también otros desenlaces secundarios, como la necesidad de reintervención, la formación de fístulas, entre otros (15). En ese orden de ideas, se documentaron 2 pacientes de 81 y 83 años de edad, en quienes se desarrolló una fístula arteriovenosa dural inmediatamente después de la embolización. De ellos, al primero se le había realizado drenaje quirúrgico del hematoma y, posteriormente, la embolización, y el segundo presentaba HSDc bilaterales, que también había tenido procedimiento quirúrgico previo. Una de las hipótesis por la cual se considera se dio fisiopatológicamente esta complicación, es que se genera una redistribución del flujo sanguíneo arterial y causa la fístula arteriovenosa (76).

Existen complicaciones reportadas con baja frecuencia, sobre todo en pacientes de edad avanzada con muchas comorbilidades. La principal es que se filtre material de embolización dentro de la arteria oftálmica o vasos vasculares del nervio facial $(0,6 \%)$, complicación que se puede manejar evitando las colaterales durante el procedimiento (70).

\section{Complicaciones}

La mortalidad intrahospitalaria ha demostrado ser baja (2\%-5\%), aun cuando puede incrementarse hasta llegar a ser del $30 \%$ a los 6 meses o un año posterior al hematoma subdural (30). Las complicaciones asociadas al drenaje de un hematoma subdural son las convulsiones (2\%), la hemorragia intracerebral $(0,7-5 \%)$, la misma recurrencia o falla del cerebro para reexpandirse $(4 \%)$, el neumoencéfalo a tensión (11\%), la infecciones y el empiema cerebral (5 $\%)$. La mortalidad es del $0 \%-8 \%$, que es mayor al someter a paciente a craneotomía, y en edades mayores a 60 años con comorbilidades (4\%) $(37,65)$. Al comparar la tasa de recurrencia, se ha evidenciado que es mayor en la craneostomía con taladro giratorio (28,1\%), luego de la craneotomía (19,4\%), y finalmente la menor tasa es reportada con la craneostomía con agujeros de trepanación (11,7\%) (64).

\section{Resultados y seguimiento}

La evolución y la mejoría neurológica de los pacientes depende no solo de condiciones intrínsecas de ellos, como la edad y las comorbilidades, sino también según los predictores de recurrencia ya enunciados y al tipo de cirugía. Se ha evidenciado que la craneostomía con agujeros de trepanación, unida a un drenaje posterior durante el posoperatorio, presenta unos desenlaces, en cuanto a la mejoría neurológica del paciente, del 84,9\%; en la craneostomía con taladro giratorio, del 93,5\%, y en la craneotomía, del $74,4 \%$. Sin embargo, la craneotomía está asociada con mayor morbilidad que las craneostomías ( $12,3 \%$ vs. $3 \%-4 \%)$, y la recurrencia del HSDc con el taladro giratorio fue mayor que con agujeros de trepanación, en los porcentajes mencionados $(62,64)$. En cuanto a la subdurostomía, se deja para ser retirada entre 24 y $48 \mathrm{~h}$ después posoperatorio. No se recomienda, en general, realizar TAC de cráneo 
simple como condición imperativa para su retiro, sino que se basa en la mejoría clínica del paciente y la temporalidad con ausencia de drenaje en el circuito cerrado de la subdurostomía, para identificar que es seguro. Para que esto se dé la posición del paciente debe ser en decúbito supino, completamente horizontal $(6,66)$.

\section{Conclusiones}

El HSDc es una patología frecuente en medicina general y neurocirugía; no obstante, a pesar de que ha sido descrito desde hace siglos, actualmente siguen los estudios para buscar el éxito en su manejo. La cirugía sigue siendo el manejo de elección; sin embargo, el manejo médico ha tenido auge en los últimos años, con estudios de medicamentos que buscan reducir la tasa de recurrencia o en casos en los cuales la cirugía no es la primera opción. Sumado a esto, la terapia endovascular ha tenido acogida en casos de recurrencia o contraindicación quirúrgica. Es necesario continuar realizando ensayos clínicos aleatorizados para poder obtener mejor evidencia de mejor calidad para la aplicabilidad de este procedimiento.

\section{Referencias}

1. Wong-Achi X, Cabrera D. Patogénesis y fisiopatología del hematoma subdural crónico. Rev Mex Neuroci. 2016. 17(4):78-85.

2. Sahyouni R, Goshtasbi K, Mahmoodi A, Tran D, Chen J. Chronic subdural hematoma: a historical and clinical perspective. World Neurosurg. 2017;108:948-53.

3. Watanabe S, Shimada H, Ishii S. Production of clinical form of chronic subdural hematoma in experimental animals. J Neurosurg. 1972;37:552-61.

4. Court J, Touchette JC, IorioMorin C, Westwick HJ, Belzile, F, Effendi, K. Embolization of the middle meningeal artery in chronic subdural hematoma: a systematic review. Clin Neurol Neurosurg. 2019;186:105464.

5. Nakaguchi $\mathrm{H}$, Tanishima $\mathrm{T}$, Yoshimasu N. Factors in the natural history of chronic subdural hematomas that influence their postoperative recurrence. J Neurosurg. 2001;95(2):256-62.

6. Abouzari M, Rashidi A, Rezaii J, Esfandiari $\mathrm{K}$, Asadollahi M, et al. The role of postoperative patient posture in the recurrrence of traumatic chronic subdural hematoma after burr-hole surgery. Neurosurgery. 2007;61(4):794-7.

7. Ohba S, Kinoshita Y, Nakagawa $\mathrm{H}$, Murakami $\mathrm{H}$. The risk factors for recurrence of chronic subdural hematoma. Neurosurg Rev. 2013;36(1):145-9.

8. Desai VR, Scranton RA, Britz GW. Management of recurrent subdural hematomas. Neurosurg Clin North Am. 2017;28(2):279-86.

9. Markwalder TM. Chronic subdural hematomas: a review. J Neurosurg. 1981;54(5):637-45.

10. Mori K, Maeda M. Surgical treatment of chronic subdural hematoma in 500 consecutive cases: clinical characteristics, surgical outcome, complications and recurrence rate. Neurol Med Chir (Tokyo). 2001;41:371-81.

11. Torihashi K, Sadamasa N, Yoshida K, Narumi O, Chin M, Yamagata S. Independent predictors for recurrence of chronic subdural hematoma: a review of 343 consecutive surgical cases. Neurosurgery. 2008;63:1125-9.

12. Castro-Rodríguez C, Román-Pena P, Arán-Echabe E, Gelabert-González M. Hematoma subdural crónico en pacientes muy ancianos. Rev Esp Geriatr Gerontol. 2016;51(6):309-16. 
13. Link, TW, Boddu, S, Paine, SM, Kamel, H, Knopman, J. Middle meningeal artery embolization for chronic subdural hematoma: a series of 60 cases. Neurosurgery. 2018;85(6):801-7.

14. Kim E. Embolization therapy for refractory hemorrhage in patients with chronic subdural hematomas. World Neurosurg. 2017;101:520-7. https://do i.org/10.1016/j.wneu.2017.02.070

15. Entezami $\mathrm{P}$, Boulos A, Paul A, Neurollahzadeh E, Dalfino J. Contrast enhancement of chronic subdural hematomas after embolization of the middle meningeal artery. Interv Neuroradiol. 2019;25(5):596-600. https://doi.org/10 $.1177 / 1591019919843354$

16. Vacca M, Argento I. Hematoma subdural crónico: una complicación frecuente. Nursing. 2019;36:16-23.

17. Edlmann E, Holl DC, Lingsma HF, Bartek J, Bartley A, Duerinck J, et al. Systematic review of current randomised control trials in chronic subdural haematoma and proposal for an international collaborative approach. Acta Neurochir (Wien). 2020;162:763-76. https://doi.org/10.10 07/s00701-020-04218-8

18. Mandai S, Sakurai M, Matsumoto Y. Middle meningeal artery embolization for refractory chronic subdural hematoma. J Neurosurg. 2000;93:686-8.

19. Killefer JA, Killefer FA, Schochet SS. The outer neomembrane of chronic subdural hematoma. Neurosurg Clin North Am. 2000;11(3):407-12.

20. Pil Ban S, Hwang G, Byon HS, Kim T, Lee SU, Ban JS, et al. Middle meningeal artery embolization for chronic subdural hematoma. Radiology. 2018;286(3):992-9. https:// doi.org/10.1148/radiol.2017170053
21. Srivatsan A, Mohanty A, Nascimiento F, Hafeez M, Srinivasan $\mathrm{V}$, Thomas A, et al. Middle meningeal artery embolization for chronic subdural hematoma: metaanalysis and systematic review. World Neurosurg. 2019;122:613-9.

22. Haldrup M, Ketharanathan B, Debrabant B, Schwartz O, Mikkelsen R, Fugleholm K, et al. Embolization of the middle meningeal artery in patients with chronic subdural hematoma: a systematic review and meta-analysis. Acta Neurochir. 2020;162:777-84.

23. Wang $\mathrm{H}$, Wang $\mathrm{C}$, Zefu L. Recurrent bilateral chronic subdural hematoma after interventional embolization combined with drilling and drainage treatment.

J Craniofac Surg. 2020 Mar/ Apr;31(2):e171-73. https://doi.org/10. 1097/SCS.0000000000006128

24. Ng S, Derraz I, Boetto J, Dargazanli $\mathrm{C}$, Poulen $\mathrm{G}$, et al. Middle meningeal artery embolization as an adjuvant treatment to surgery for symptomatic chronic subdural hematoma: a pilot study assessing hematoma volume resorption. J Neurointervent Surg. 2020 Jul;12 (7):695-699. https://doi.org /10.1136/neurintsurg-2019-015421

25. Fiorella D, Arthur, AS. Middle meningial artery embolization for the management of chronic subdural hematoma. J Neurointervent Surg. 2019 Sep;11(9):912-5. https:// doi.org/10.1136/

neurintsurg-2019-014730

26. Yeomans J. Nuerosurgical surgery. Toronto: WB Saunders Company; 1973. p. 963-5.

27. Markwalder TM, Steinsiepe KF, Rohner M, Reichenbach W, Markwalder $\mathrm{H}$. The course of chronic subdural hematoma after burrhole craniotomy and closed-system drainage. J Neurosurg. 1981;55:390-6. 
28. Sun TF, Boet R, Poon WS. Nonsurgical primary treatment of chronic subdural haematoma: preliminary results of using dexamethasone. $\mathrm{Br} \mathrm{J}$ Neurosurg. 2005;19(4):327-33. https:// doi.org/10.1080/02688690500305332

29. Teale E, Iliffe S, Young J. Subdural haematoma in the elderly. BMJ [Imagen internet]. 2014;348:36-8.

30. Siddiqui F, Bekker S, Qureshi A. Neuroimaging of hemorrhage and vascular defects. Neurotherapeutics. 2011;8:28-38.

31. Gálvez M, Bravo E, Rodríguez P, Farías M, Cerda J. Características de las hemorragias intracraneanas espontáneas en TC y RM. Rev Chil Radiol. 2007;13:12-25.

32. Okuma Y, Hirotsune N, Sato Y, Tanabe T, Muraoka K, Nishino S. Midterm follow-up of patients with middle meningeal artery embolization in intractable chronic subdural hematoma. World Neurosurg. 2019;126:e671-8.

33. Stanisic M, Lund-Johasen M, Mahesparan R. Treatment of chronic subdural hematoma by burr-hole craniostomy in adults: influence of some factors on postoperative recurrence. Acta Neuriochir. 2005;147(12):1249-57.

34. Chihara $\mathrm{H}$, Imamura $\mathrm{H}$, Ogura $\mathrm{T}$, Adachi $\mathrm{H}$, Imai $\mathrm{Y}$, Sakai N. Recurrence of a refractory chronic subdural hematoma after middle meningeal artery embolization that required craniotomy. NMC Case Rep J. 2013;1(1):1-5. https://doi.org/10.2176/ nmccrj.2013-0343

35. Tsukamoto Y, Oishi M, Shinbo J, Fujil Y. Transarterial embolisation for refractory bilateral chronic subdural hematomas in a case with dentatorubral-pallidoluysian atrophy. Acta Neurochir. 2011;153:1145-7.
36. Soleman J, Nocera F, Mariani L The conservative and pharmacological management of chronic subdural haematoma. Swiss Med Wkly. 2017;147:w14398.

37. Fuenmayor D, Quintanilla C, Olmedo J, Rodríguez L. Hematoma subdural crónico: tratamiento. Recimundo. 2020;4:184-98.

38. Janowski M, Kunert P. Intravenous fluid administration may improve post operative course of patients with chronic subdural hematoma: a retrospective study. PloS One. 2012;7(4):e35634. https://doi.org/10.1 371/journal.pone.0035634

39. Thotakura, AK, Marabathina NR. nonsurgical treatment of chronic subdural hematoma with steroids. World Neurosurg. 2015;84(6):1968-72.

40. Bender MB, Christoff N. Nonsurgical treatment of subdural haematomas. Arch Neurol. 1974, 31:73-9.

41. Chan DY, Sun TF, Poon WS. Steroid for chronic subdural hematoma? A prospective phase IIB pilot randomized controlled trial on the use of dexamethasone with surgical drainage for the reduction of recurrence with reoperation. Chin Neurosurg J. 2015;1:2.

42. Hutchinson P, Edlmann E, Bulters $\mathrm{D}$, Zolnourian A, Holton $\mathrm{P}$, et al. Trial of dexamethasone for chronic subdural hematoma. N Engl J Med. 2020;383:2616-27.

43. Weigel R, Hohenstein A, Schlickum L, Weiss C, Schilling L. Angiotensin converting enzyme inhibition for arterial hypertension reduces the risk of recurrence in patients with chronic subdural hematoma possibly 
by an antiangiogenic mechanism. Neurosurgery. 2007;61:188-92.

44. Poulsen FR, Munthe S, Soe M, Halle B. Perindropril and residual chronic subdural hematoma volumes six weeks after burr hole surgery: a randomized trial. Clin Neurol Neurosurg. 2014;123:4-8.

45. Neidert MC, Schmidt T, Mitova T, Fierstra J, Bellut D, Regli L, et al. Preoperative angiotensin converting enzyme inhibitor usage in patients with chronic subdural hematoma: associations with presentation and clinical outcome. J Clin Neurosci. 2016;28:82-6.

46. Thotakura AK, Marabathina NR. The role of medical treatment in chronic subdural hematoma. Asian J Neurosurg. 2018;13(4):976-83.

47. Vujkovac B, Sabovic M. Treatment of subdural and intracerebral haematomas in a haemodialysis patient with tranexamic acid. Nephrol Dial Trasplant. 2000;15:107-9.

48. Kageyama H, Toyooka T, Tsuzuki $\mathrm{N}$, Oka K. Nonsurgical treatment of chronic subdural hematoma with tranezamic acid. J Neurosurg. 2013;119:332-7.

49. Tanweer O, Frisoli FA, Bravate C, Harrison G, Pacione D, Kondziolka D, et al. Tranexamic acid for treatment of residual subdural hematoma after bedside twist-drill evacuation. World Neurosurg. 2016;91:29-33.

50. Ioro-Morin C, Blanchard J, Richer M, Mathieu D. Tranexamic Acid in Chronic Subdural Hematomas (TRACS): study protocol for a randomized controlled trial. Trials. 2016 May 5;17 (1):235. https://doi.org/ 10.1186/s13063-016-1358-5

51. Hirashima Y, Endo S, Kato R, Ohmori $T$, nagahori $T$, Nishijima $\mathrm{M}$, et al. Effect of platelet- activating factor receptor antagonista, etizolam, on resolution of chronica subdural ehmatoma: a prospective study to investigate use as conservative therapy. Neurol Med Chir (Tokyo). 2005;45:621-6. https://doi.org/10.2176 /nmc.45.621

52. Seok Lee K. How to treat chronic subdural hematoma? Past and now. J Korean Neurosurg Soc. 2019;62(2):144-52.

53. Suzuki J, Takaku A. Nonsurgical treatment of chronic subdural hematoma. J Neurosurg. 1970;33:548-53.

54. Gjerris F, Schmidt K. Chronic subdural hematoma: surgery or mannitol treatment. J Neurosurg. 1974;40(5):639-42.

55. Wang D, Li T, Tian Y Wang S, Jin $\mathrm{C}$, Wei $\mathrm{H}$, et al. Effects of atorvastatina on chronic subdural hematomas: a preliminary report from three medical centers. J Neurol Sci. 2014;336:237-42.

56. Bullock MR, Chesnut R, Ghajar J, Gordon D, Heartl R, Newell DW, Wilberger JE. Surgical management of acute subdural hematomas. Neurosurgery. 2006;58(3):S2-16.

57. Greenberg MS. Handbook of neurosurgery. 8. ${ }^{-}$ed. Tampa, Florida: Thieme; 2016.

58. López A, Blanco C, Lara $\mathrm{R}$, Moscote L. Hematoma subdural crónico: atención neurointensiva. Revmie. 2018;17:86-91.

59. Kolias A, Chari A, Santarius T, Hutchinson P. Chronic subdural haematoma: modern management and emerging therapies. Nat Rev Neurol. 2014;10:570-8.

60. Ramnarayan R, Arulmurugan B, Wilson P, Nayar R. Twist drill craniostomy with closed drainage for chronic subdural haematoma in the 
elderly: an effective method. Clin Neurol Neurosurg. 2008;110:774-8.

61. Jong-Lee S, Hwang S, Bin S. Twist-drill or burr hole craniostomy for draining chronic subdural hematomas: how to choose it for chronic subdural hematoma drainage. Korean J Neurotrauma. 2016;12:107-11.

62. Santarius T, Kirkpatrick P, Ganesan $\mathrm{D}$, Chia H, Jalloh I, et al. Use of drains versus no drains after burrhole evacuation of chronic subdural haematoma: a randomised controlled trial. Lancet. 2009;374:1067-73.

63. Ven Der Veken, Duerink J, Buyl R, Van Rompaey K, Herregodts P, D’Haens J. Mini-craniotomy as the primary surgical intervention for the treatment of chronic subdural hematoma: a retrospective analysis. Acta Neurochir. 2014;156:981-7.

64. Ducruet A, Grobelny B, Zacharis B, Hickman Z, DeRosa P, Andersen K, et al. The surgical management of chronic subdural hematoma. Neurosurg Rev. 2012;35:155-69.

65. Moreno Huertas CE. Factores asociados a recurrencia en hematomas subdurales crónicos: un estudio de cohorte retrospectiva en el hospital universitario mayor [internet]. Bogotá: Universidad del Rosario; 2019. Disponible en: http://repository.urosari o.edu.co/handle/10336/20018

66. Kwon TH, Park YK, Lim DJ, Cho TH, Chung YG, Chung HS, Suh JK. Chronic subdural hematoma: evaluation of the clinical significance of postoperative drainage volume. J Neurosurg. 2000;93(5):796-9. https://d oi.org/10.3171/jns.2000.93.5.0796

67. Gazzeri R, Galarza M, Neroni M, Canova A, Refice GM, Esposito S. Continuous subgaleal suction drainage for the treatment of chronic subdural haematoma. Acta Neurochir. 2007;149(5):487-93.

68. Hostalot-Panisello C, CarrascoGonzález A, Bilbao-Barandica G, Pomposo-Gaztelu I, Garibi JM. Hematoma subdural crónico: presentación y actitudes terapéuticas. Rev Neurol. 2002;35(2):123-7.

69. Senkichi J. Embolic agents. Intervencionism. 2016;16(1):16-26.

70. Kan P, Maragkos G, Srivatsan A, Srinivasan V, Johnson J, Burkhardt JK, Robinson $\mathrm{T}$, et al. Middle meningeal artery embolization for chronic subdural hematoma: a multi-center experience of 154 consecutive embolizations. Neurosurgery. 2020;88(2):268-77.

71. Rajah G, Tso, MK, Dossani R, Vakharia K, Siddiqui AH. Transradial embolization of the left middle meningeal artery and accessory middle meningeal artery for treatment of subacute-chronic subdural hematoma. J NeuroIntervent Surg Epub. 2020 Apr;12(4):436. https://doi.org/10.1136 /neurintsurg-2019-015493

72. Waqas M, Vackhari K, Weimer P, Hashmi E, Davies JM, Siddiqui AH. Safety and effectiveness of embolization for chronic subdural hematoma: systematic review and case series. World Neurorug. 2019;126:228-36. https://doi.org/10.10 16/j.wneu.2019.02.208

73. Hirai S, Ono J, Odaki M, Serizawa T, Nagano O. Embolization of the middle meningeal artery for refractory chronic subdural haematoma: usefulness for patients under anticoagulant therapy. Interv Neuroradiol. 2004;10 Suppl 2:101-4. https://doi.org/10.1177/15910 $199040100 S 218$

74. Chihara $\mathrm{H}$, Imamura $\mathrm{H}$, Ogura $\mathrm{T}$, Adachi $\mathrm{H}$, Imai $\mathrm{Y}$, Sakai $\mathrm{N}$. 
Recurrence of a refractory chronic subdural hematoma after middle meningeal artery embolization that required craniotomy. NMC Case Rep J. 2014;1(1):1-5. https://doi.org/10.2176/ nmccrj.2013-0343

75. Sattur M, Spiotta A. Anomalous "middle" meningeal artery from basilar artery and implications for neuroendovascular surgery: case report and review of literature. World Neurosurg. 2020;133:84-9.

76. Piergallini L, Dargazanli C, Derraz I, Costalat V. Immediate development of dural arteriovenous fistula after middle meningeal artery embolization: first angiographic demonstration. World Neurosurg. 2019;128:606-10. https://doi.org/10.10

16/j.wneu.2019.04.246 